\title{
Relationship Between Blood Pressure, Cerebral Electrical Activity, Cerebral Fractional Oxygen Extraction, and Peripheral Blood Flow in Very Low Birth Weight Newborn Infants
}

\author{
SURESH VICTOR, ANTHONY G. MARSON, RICHARD E. APPLETON, MARGARET BEIRNE, AND A. MICHAEL WEINDLING
}

\begin{abstract}
Division of Child Health [S.V., M.W.], University of Liverpool, Liverpool Women's Hospital, Neonatal Unit, Liverpool, UK, L8 7SS; Division of Neurological Science [A.G.M.], University of Liverpool, Clinical Sciences Centre, Fazakerley, Liverpool, UK, L9 7LJ; Department of Neurology [R.E.A., M.B.], Royal Liverpool Children's Hospital NHS Trust (Alder Hey), The Roald Dahl EEG Unit,
\end{abstract} Liverpool, UK, L12 $2 A P$

\begin{abstract}
There is uncertainty about the level of systemic blood pressure required to maintain adequate cerebral oxygen delivery and organ integrity. This prospective, observational study on 35 very low birth weight infants aimed to determine the mean blood pressure (MBP) below which cerebral electrical activity, peripheral blood flow (PBF), and cerebral fractional oxygen extraction (CFOE) are abnormal. Digital EEG, recorded every day on the first $4 \mathrm{~d}$ after birth, were analyzed a) by automatic spectral analysis, b) by manual measurement of interburst interval, and c) qualitatively. CFOE and PBF measurements were performed using near-infrared spectroscopy and venous occlusion. MBP was measured using arterial catheters. The median (range) of MBP recorded was $32 \mathrm{~mm} \mathrm{Hg}(16-46)$. The EEG became abnormal at MBP levels below $23 \mathrm{~mm} \mathrm{Hg}$ : a) the relative power of the delta $(0.5-3.5 \mathrm{~Hz})$ frequency band was decreased, b) interburst intervals were prolonged, and c) all four qualitatively abnormal EEG (low amplitude and prolonged interburst intervals) from four different patients were recorded below this MBP level. The only abnormally high CFOE was measured at MBP of $20 \mathrm{~mm} \mathrm{Hg}$. PBF decreased at MBP levels between 23 and $33 \mathrm{~mm} \mathrm{Hg}$. None of the infants in this study developed cystic periventricular leukomalacia. One infant (MBP, $22 \mathrm{~mm} \mathrm{Hg}$ ) developed ventricular dilatation after intraventricular hemorrhage. The EEG and CFOE remained normal at MBP levels above $23 \mathrm{~mm} \mathrm{Hg}$. It would appear that cerebral perfusion is probably maintained at MBP levels above $23 \mathrm{~mm} \mathrm{Hg}$. (Pediatr Res 59: 314-319, 2006)
\end{abstract}

$\mathrm{S}^{\mathrm{c}}$ everal authors have described an association between systemic hypotension in premature infants and neurologic morbidity (1-3), and, in some centers, clinical practice is to support blood pressure by inotropes and volume expanders when the MBP level falls below $30 \mathrm{~mm} \mathrm{Hg}$. The likely mechanism by which hypotension causes neurologic damage is by diminished oxygen delivery through decreased cerebral perfusion. However, the relationship between MBP and cerebral blood flow is unclear in premature infants $(4-8)$. Some authors have argued that cerebral blood flow is pressure passive and dependent on MBP in infants between 28 and 39

Received May 5, 2005; accepted September 19, 2005.

Correspondence: Suresh Victor, MBBS, MRCPCH, Clinical Research Fellow, Neonatal Intensive Care Unit, Liverpool Women's Hospital, Crown Street, Liverpool, L8 7SS, UK; e-mail: svictor@liverpool.ac.uk

Supported by Newborn Appeal, Liverpool Women's Hospital, Liverpool, UK L87SS.

DOI: 10.1203/01.pdr.0000199525.08615.1f wk gestation $(5,7)$. Others, who have studied infants between 24 and 34 wk gestation, observed that cerebral blood flow is independent of MBP $(4,8)$. Furthermore, the critical level of MBP at which cerebral perfusion becomes compromised has not been clearly determined.

In spite of a lack of evidence linking systemic hypotension to brain damage in very low birth weight infants, it is well known that older subjects lose consciousness when MBP falls to a seriously low level. A change in the level of consciousness may not be recognized in sick newborn infants who are heavily sedated while being ventilated, but it may be associated with recognizable changes in the EEG pattern. Using a cerebral function monitor, Greisen et al. (9) showed that reduced blood flow to the neonatal brain correlated with decreased amplitude of the EEG. There have been no other studies that have investigated the relationship between background cerebral electrical activity and hemodynamic variables in very low birth weight infants. Nevertheless, the EEG pattern of infants between 26 and $30 \mathrm{wk}$ gestation has been well characterized (10). It is markedly discontinuous and consists of long periods of quiescence, called interburst intervals, interspersed with bursts of high-voltage activity of mixed frequency. With increasing gestation, the interburst intervals decrease and the record becomes more continuous (11). Abnormal background EEG activity characterized by prolonged interburst intervals has been linked to death and adverse neurologic outcome in preterm babies (12).

The largest portion of the brain oxygen consumption is used to maintain transmembrane ion gradient (13). Decreased cerebral oxygen delivery therefore leads to a sequence of alterations in the transmembrane electrochemical gradients, and hence in surface EEG patterns (13). Tissue oxygen deprivation is determined by the dynamic relationship between oxygen supply and oxygen demand. A balance between cerebral oxygen delivery and utilization would therefore be expected to

Abbreviations: CFOE, cerebral fractional oxygen extraction; MBP, mean blood pressure; PBF, peripheral blood flow; $\mathbf{P}_{\mathbf{9 0}}$, 90th centile 
provide an indication of this energy balance at the time of EEG recording (14,15). At lower levels of MBP (23-27 mm $\mathrm{Hg}$ ), PBF would be expected to diminish (16).

The purpose of this study was to determine the relationship between MBP, cerebral electrical activity, CFOE, and PBF in premature newborn infants, with an intention of establishing the level of MBP at which management of hypotension is to be instituted.

\section{METHODS}

This was a prospective observational study performed on 35 very low birth weight infants of less than $30 \mathrm{wk}$ gestation born at Liverpool Women's Hospital. Measurements were performed every day on the first $4 \mathrm{~d}$ after birth. Ethical approval was obtained from the Liverpool Children's Research Ethics Committee and informed parental consent was obtained. An upper gestational age limit of $30 \mathrm{wk}$ was chosen as sleep-wake cycling is typically not seen below this gestation (17). Infants with serious intraventricular hemorrhage (defined as any hemorrhage extending beyond the germinal matrix) on the first $2 \mathrm{~d}$ after birth were excluded from the study.

$\boldsymbol{E E G}$. Digital EEG and ECG recordings were performed for 75 min using a Micromed 16-channel system (Micromed Electronics Ltd., UK). Six electrodes were placed on the frontopolar (Fp1, Fp2), central (C3, C4), and occipital $(\mathrm{O} 1, \mathrm{O} 2)$ positions bilaterally according to the International 10-20 System (18), A reference electrode was placed at the vertex (Cz). Skin impedance of $<2 \mathrm{k} \Omega$ was maintained for all recordings. A sampling rate of $256 \mathrm{~Hz}$ was used for digitization.

The EEG was analyzed qualitatively and quantitatively. M.B. and R.A., both experienced at reporting neonatal and pediatric EEG, undertook the qualitative reporting. They were blind to MBP and cranial ultrasound scan findings. The EEG was displayed on a computer screen as four bipolar channels (Fp1 - C3, C3 - O1, Fp2 - C4, and C4-O2) using a high-pass filter of $0.3 \mathrm{~Hz}$, a low-pass filter of $70 \mathrm{~Hz}$, a notch filter of $50 \mathrm{~Hz}$, a base time of $10 \mathrm{~s}$, and a gain of $100 \mu \mathrm{V}$. The EEG recordings were analyzed for degree of discontinuity, amplitude, presence of abnormal transients, and synchrony.

Quantitative analysis of EEG was by a) spectral analysis and b) manual calculation of the interburst interval. To calculate the interburst intervals, gross artifacts (activity with no identifiable normal EEG activity) were identified by eye and removed. The interburst interval was defined as a period between electrical bursts during which activities were lower than $30 \mu \mathrm{V}$ in all leads and calculated manually (19). The 90th centile $\left(\mathrm{P}_{90}\right)$ for interburst interval was then calculated for the first $60 \mathrm{~min}$ of artifact-free recording for each baby. The normal range (10th to 90th centile) of $\mathrm{P}_{90}$ interburst interval in infants between 24 and $30 \mathrm{wk}$ gestation is $7-25 \mathrm{~s}$ during the first $2 \mathrm{~d}$ after birth (20).

Spectral analysis using Fast Fourier transformation was performed using the manufacturer's software (Micromed) and is described in detail elsewhere (20). The 75 min of EEG recorded from six monopolar channels (Fp1, Fp2, $\mathrm{C} 3, \mathrm{C} 4, \mathrm{O} 1$, and $\mathrm{O} 2$ ) was subjected to spectral analysis. The spectrum was subdivided into delta $(0.5-3.5 \mathrm{~Hz})$, theta $(4-7.5 \mathrm{~Hz})$, alpha $(8-12.5 \mathrm{~Hz})$, and beta $(13-30 \mathrm{~Hz})$ frequency bands. The absolute power of a frequency band was defined as the integral of the power values within the frequency range and expressed as $\mu \mathrm{V}^{2}$. The relative power (RP) of a frequency band was defined as the ratio of the absolute power of that frequency band to the total power of the EEG signal and expressed as a percentage. The absolute and relative powers of each frequency band were calculated for every 10-s epoch. Gross artifacts (activity with no identifiable normal EEG activity) were identified by eye and removed manually in 10-s epochs. The first $60 \mathrm{~min}$ of artifact-free EEG was then used to calculate the median absolute and the median relative powers of each frequency band. For very low birth weight infants, the relative power of the delta frequency band is the most discriminatory and repeatable (coefficient of repeatability of $8 \%$ ) spectral measurement (20). The normal range (10th to 90th centile) of the relative power of the delta frequency band in infants between 24 and $30 \mathrm{wk}$ gestation is $62-82 \%$ during the first $2 \mathrm{~d}$ after birth (20).

CFOE. CFOE measurements were made during each EEG recording. Cerebral venous oxygen saturation $\left(\mathrm{CSvO}_{2}\right)$ was measured by partial jugular venous occlusion and the Hamamatsu NIRO 500 (Hamamatsu Corp., Bridgewater, NJ) with a pulse oximeter in beat-to-beat mode (Datex-Ohmeda, Madison, WI) $(14,15)$. The mean of five partial jugular venous occlusions made over a 5-10 min period was taken (15). Cerebral arterial oxygen saturation $\left(\mathrm{CSaO}_{2}\right)$ was assumed to be equal to peripheral arterial oxygen saturation. $\mathrm{CFOE}$ was calculated using the formula: $\mathrm{CFOE}=\mathrm{CSaO}_{2}-\mathrm{CSvO}_{2} / \mathrm{CSaO}_{2}(15)$. The mean $\pm \mathrm{SD}$ of $\mathrm{CFOE}$ in clinically stable infants between 27 and 31 wk gestation was $0.29 \pm 0.06$ (14). The coefficient of repeatability of CFOE measurements was 0.05 .

$\boldsymbol{P B F}$. Measurements of PBF were made during each EEG recording, using a method that has been described in detail elsewhere (21). The Hamamatsu NIRO 500 and a pulse oximeter in beat-to-beat mode (Datex-Ohmeda) were used to measure total tissue $\mathrm{Hb}$ concentration $(\Delta \mathrm{HbT})$ in the forearm. Hb flow was then calculated by the slope of a line through the $\Delta \mathrm{HbT}$ values during the first $2 \mathrm{~s}$ of occlusion using a least squares method. $\mathrm{PBF}(\mathrm{mL} / 100 \mathrm{~mL} / \mathrm{min})$ was calculated by dividing $\mathrm{Hb}$ flow by venous $\mathrm{Hb}$ concentration. The normal range (minimum-maximum) of $\mathrm{PBF}$ in infants between 28 and 32 wk gestation is $6.1-13.4 \mathrm{~mL} / 100 \mathrm{~mL} / \mathrm{min}(21)$.

Clinical data. Demographic details and the use of inotropes and sedatives were recorded. MBP measurements were recorded from an indwelling arterial catheter every 4 min during each EEG recording. The mean of these measurements was used for analysis. Arterial blood gas measurement was performed midway through the EEG recording. Histologic evidence of chorioamnionitis (defined as presence of polymorphonuclear leukocytes in the chorion and amnion) was also recorded.

Clinical management. MBP monitoring was done by Nova-dome pressure transducer (Micromed) attached to the indwelling arterial catheter. The attending physician, who was not a member of the research group, determined the management of hypotension. It was generally aimed to keep the MBP above the 10th centile for the birth weight (22). This was achieved by volume expansion using blood transfusion or normal saline or inotropes such as dopamine, dobutamine, or hydrocortisone used singly or in combination. All infants who received morphine were prescribed a dose of $20 \mu \mathrm{g} / \mathrm{kg} / \mathrm{h}$.

Follow-up measurements. Cranial ultrasound scans were performed on all infants on the first $4 \mathrm{~d}$, after birth and repeated at regular fortnightly intervals before discharge.

Statistical analysis. The purpose of this study was to investigate the cerebrovascular effects of hypotension. This invariably occurred during the first $48 \mathrm{~h}$. Therefore, the measurements of PBF, CFOE and EEG made at the lowest MBP during this period [within a preset range of arterial carbon dioxide partial pressure between 35 and $50 \mathrm{~mm} \mathrm{Hg}$ (20)] were selected for each infant for further analysis.

The EEG was analyzed in relation to MBP. For analysis of the quantitative EEG results, univariate analysis was performed with the relative power of each frequency band and interburst interval duration as the dependent variables with MBP as the independent variable. For significant univariate relationships, a stepwise regression using gestation, MBP, dopamine infusion rate $(\mu \mathrm{g} / \mathrm{kg} / \mathrm{h})$ at the time of recording, $\mathrm{pH}$, and $\mathrm{PCO}_{2}$ as predictor variables was performed to rule out any confounding factors.

Curve fitting was done where appropriate using the F-test provided by SPSS version 10 (SPSS Inc., Chicago, IL). Linear, quadratic, and cubic regression curves were fitted. F-ratios were calculated to determine which model provided the best fit.

Regression curves with $95 \%$ confidence intervals were plotted on graphs that also included the normal ranges for the relative power of delta band and for the $\mathrm{P}_{90}$ interburst interval. The highest MBP at which the $95 \%$ confidence intervals of the regression curve intercepted the normal range for the relative power of delta band or the $\mathrm{P}_{90}$ interburst interval was chosen as the critical point of MBP at which EEG became abnormal

The demography and condition of the infants with qualitatively normal and abnormal EEG records were compared using the Mann-Whitney $U$ test (Table $1)$.

\section{RESULTS}

The demography and condition of infants at the time of EEG recordings are described in Table 1. Thirty-four infants were ventilated at the time of measurement. All infants had normal blood glucose concentrations at the time of recording. The daily measurements of peripheral blood flow, CFOE, MBP, and EEG have been summarized in Table 2.

The lowest MBP during the first $48 \mathrm{~h}$ for each baby was related to the corresponding EEG, CFOE, and PBF measurements.

The EEG was recorded in all cases. Using qualitative analysis, 31 EEG records were normal: all four abnormal EEG were recorded at MBP levels below $23 \mathrm{~mm} \mathrm{Hg}$. The abnormal qualitative reports and their corresponding levels of MBP are described in Table 3. Examples of EEG traces from three 
Table 1. Demography and condition of infants in the study group and the four infants with abnormal EEG records

\begin{tabular}{|c|c|c|c|c|}
\hline & All infants $(n=35)$ & Normal EEG $(n=31)$ & Abnormal EEG $(n=4)$ & $p$ Value \\
\hline Gestation (wk) & $27(23-30)$ & $27(23-30)$ & $27(24-28)$ & 0.979 \\
\hline Birth weight $(\mathrm{g})$ & $935(470-1495)$ & $935(470-1495)$ & $857(534-1160)$ & 0.604 \\
\hline IUGR (BW < 10th centile) & 9 & 8 & 1 & 0.973 \\
\hline Chorioamnionitis (no. of infants) & $6(n=25)$ & $6(n=22)$ & $0(n=3)$ & 0.309 \\
\hline Apgar at $5 \mathrm{~min}$ & $8(4-10)$ & $8(4-10)$ & $8(5-10)$ & 0.674 \\
\hline Cord base deficit (mmol/L) & $3.5(-1.3$ to 9.6$)$ & $3.5(-1.3$ to 9.6$)$ & $3.3(1-5.9)$ & 0.938 \\
\hline Arterial $\mathrm{Po}_{2}(\mathrm{~mm} \mathrm{Hg})$ & $59(35-143)$ & $59(35-143)$ & $71(40-87)$ & 0.534 \\
\hline Arterial $\mathrm{pH}$ & $7.33(7.23-7.45)$ & $7.33(7.27-7.45)$ & $7.31(7.23-7.37)$ & 0.251 \\
\hline Arterial $\mathrm{PCO}_{2}(\mathrm{~mm} \mathrm{Hg})$ & $42(36-49)$ & $42(36-49)$ & $43(38-46)$ & 0.776 \\
\hline CRIB score & $3(0-17)$ & $3(0-17)$ & $8(2-14)$ & 0.177 \\
\hline MBP (mm Hg) & $32(16-46)$ & $33(20-46)$ & $22(16-22)$ & $<0.001$ \\
\hline
\end{tabular}

Values are median (range), unless stated otherwise. $p$ Values were obtained by comparing infants with normal and abnormal EEG using Mann-Whitney $U$ test.

Table 2. Changes in MBP, CFOE, PBF, and relative power of EEG delta frequency band and EEG interburst interval during the first $4 d$ after birth

\begin{tabular}{lcccc}
\hline & \multicolumn{1}{c}{ Day 1 } & Day 2 & Day 3 & Day 4 \\
\hline MBP (mm Hg) & $32(16-46)[35]$ & $37(20-51)[35]$ & $41(27-56)[24]$ & $42(27-57)[16]$ \\
CFOE & $0.33(0.25-0.43)[21]$ & $0.28(0.14-0.49)[23]$ & $0.3(0.2-0.49)[19]$ & $0.3(0.2-0.35)[14]$ \\
PBF (mL/100 mL/min) & $4.2(2.2-12.8)[14]$ & $6.1(2.7-25.3)[16]$ & $6.4(1.4-19.2)[14]$ & $6.5(3.1-23.1)[9]$ \\
RP delta (\%) & $69(20-80)[35]$ & $73(60-87)[35]$ & $78(66-88)[35]$ & $78(58-89)[27]$ \\
P90 IBI (s) & $15(7-53)[35]$ & $12(6-31)[35]$ & $10(5-16)[35]$ & $9(3-16)[27]$ \\
\hline
\end{tabular}

Values are median (range) [no.].

Table 3. Qualitative EEG reports and cranial ultrasound scan findings in the four infants with abnormal EEG

\begin{tabular}{|c|c|c|c|c|c|c|c|}
\hline Case no. & $\begin{array}{l}\text { Age } \\
\text { (h) }\end{array}$ & $\begin{array}{c}\mathrm{MBP} \\
(\mathrm{mm} \mathrm{Hg})\end{array}$ & $\begin{array}{l}\text { RP delta } \\
(\%)\end{array}$ & $\begin{array}{c}\text { P90 } \\
\text { IBI (s) }\end{array}$ & EEG report & CFOE & Cranial ultrasound \\
\hline \multirow[t]{3}{*}{1} & 13 & 16 & 20 & 33 & $\downarrow$ Amplitude, $\uparrow$ interburst interval & & Subependymal bleed \\
\hline & 35 & 30 & 73 & 15 & Normal & & Subependymal bleed \\
\hline & 86 & 41 & 88 & 6 & Normal & & Subependymal bleed \\
\hline \multirow[t]{2}{*}{2} & 13 & 22 & 75 & 9 & Slowing,$+ \downarrow$ amplitude, seizure + & & Normal \\
\hline & 34 & 34 & 64 & 26 & $\uparrow$ Interburst interval & 0.18 & Subependymal bleed \\
\hline \multirow[t]{3}{*}{3} & 7 & 22 & 56 & 51 & $\uparrow$ Interburst interval & 0.31 & Normal \\
\hline & 24 & 38 & 80 & 28 & $\uparrow$ Interburst interval & 0.18 & Normal \\
\hline & 48 & 42 & 71 & 11 & Normal & 0.32 & Normal \\
\hline \multirow[t]{3}{*}{4} & 14 & 22 & 59 & 29 & $\downarrow$ Amplitude, $\uparrow$ interburst interval & & Normal \\
\hline & 38 & 26 & 71 & 15 & $\downarrow$ Amplitude, improved & & Subependymal bleed \\
\hline & 52 & & 87 & 9 & $\downarrow$ Amplitude, improved & & Subependymal bleed \\
\hline
\end{tabular}

IVH, intraventricular hemorrhage.

different infants at different levels of MBP are shown in Figure 1. Only MBP was significantly different between the two groups (Table 1). Only one normal EEG was recorded at MBP levels below $23 \mathrm{~mm} \mathrm{Hg}$ and clinical details of this infant (Case No. 5) are described below.

Exploration of the relationship between the relative power of each EEG frequency band and MBP showed that only the relative power of the delta frequency band had a significant relationship with MBP. The best-fit curve was a cubic regression curve (Fig. 2). The F-ratio comparing the linear model with the quadratic model was $14.5(p<0.001)$, and the F-ratio comparing the quadratic model with the cubic model was 14 $(p<0.001)$. The highest point of intercept between the 95\% confidence intervals of the cubic regression curve and the lower limit of the normal range of the relative power of the delta band was at $23 \mathrm{~mm} \mathrm{Hg}$ (Fig. 2). Three infants had abnormally low $(<10$ th centile) relative power of the delta band, resulting in leverage of the regression curve by these three points. Stepwise regression confirmed the relationship between MBP and the relative power of the delta frequency band.

Univariate analysis between the $\mathrm{P}_{90}$ interburst interval and MBP was statistically significant (Pearson's correlation: $-0.501 ; p=0.002)$. The curve of best fit was a linear regression model (Fig. 3). The highest point of intercept between 95\% confidence intervals of the linear regression curve and the upper limit of the normal range of the interburst interval was at $23 \mathrm{~mm} \mathrm{Hg}$ (Fig. 3). Only three infants had abnormally prolonged interburst intervals, resulting in lever- 
A

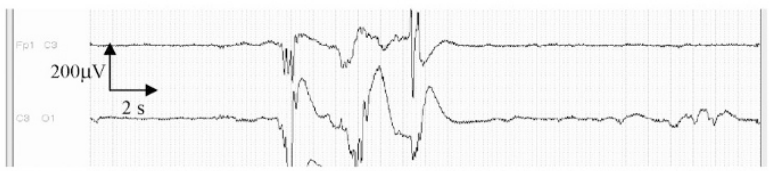

B

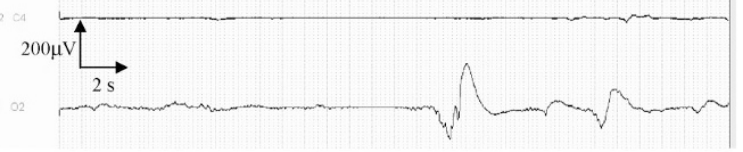

C

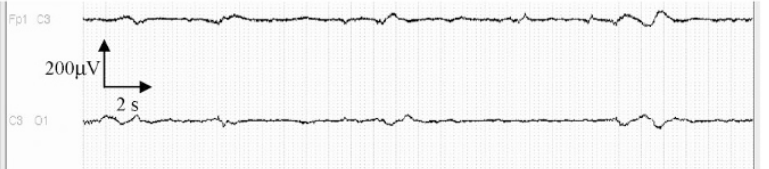

Figure 1. EEG traces from three infants at different levels of MBP. (A) Trace 1: gestation: 24 wk; age: 6 h; MBP: 26 mm Hg. (B) Trace 2: gestation: 24 wk; age: $14 \mathrm{~h}$; MBP: $22 \mathrm{~mm} \mathrm{Hg}$. (C) Trace 3: gestation: 27 wk; age: $13 \mathrm{~h}$; MBP: $16 \mathrm{~mm} \mathrm{Hg}$.

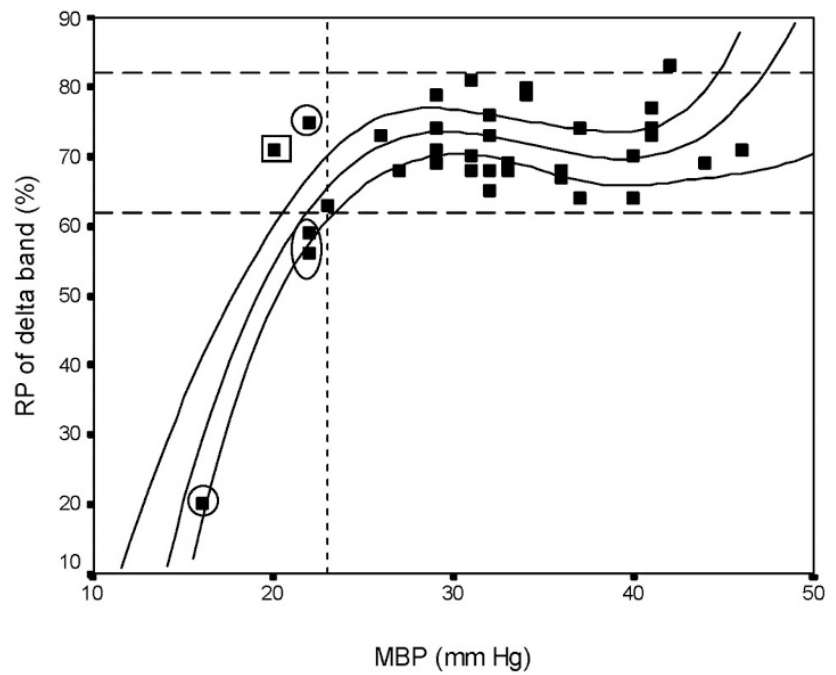

Figure 2. Relationship between MBP and the relative power (RP) of the delta band, showing line of best fit with 95\% confidence interval $(n=35$; Rsq $=$ 0.627; $p<0.001$ ). The horizontal dotted lines indicate normal range of the relative power of the delta band (10th-90th centile). Vertical dotted line indicates the point of intercept. Abnormal EEG records are circled. Square indicates an infant where the CFOE was abnormal (0.44).

age of the regression line by these three points. Stepwise regression confirmed the relationship between MBP and interburst interval.

CFOE was measured in 19 infants. Of the four infants with abnormal EEG, only one infant had CFOE (value: 0.31) measured. Loss of CFOE measurements was due to equipment failure and difficulty in gaining access to patients with severe hypotension in whom clinical management took priority. Eighteen infants had normal CFOE (median, 0.31; range, $0.25-0.40$ ). Only one infant had an abnormally high CFOE (0.44). This infant (case no. 5) is described in detail below. CFOE did not show a statistically significant relationship with MBP (Spearman's rho: $-0.31, p=0.19, n=19$ ).

$\mathrm{PBF}$ was measured in 14 infants (median, $5.2 \mathrm{~mL} / 100$ $\mathrm{mL} / \mathrm{min}$; range, 2.2-12.8). None of the infants with abnormal EEG and MBP below $23 \mathrm{~mm} \mathrm{Hg}$ had PBF measurements. The

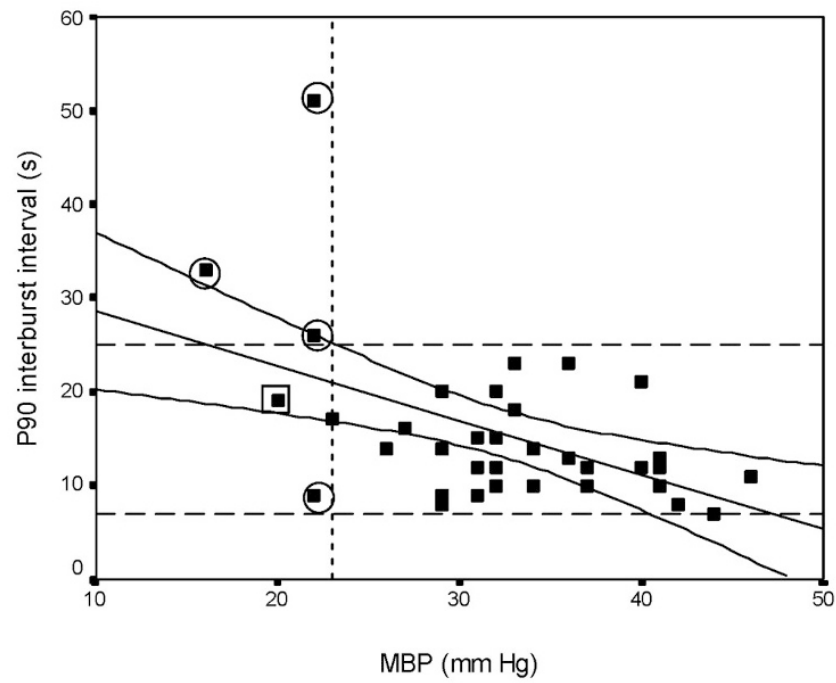

Figure 3. Relationship between MBP and the 90th centile of interburst interval, showing line of best fit with $95 \%$ confidence interval $(n=35$; Rsq $=0.251 ; p=0.002$ ). The horizontal dotted lines indicate normal range of the $\mathrm{P}_{90}$ interburst interval (10th-90th centile). Vertical dotted line indicates the point of intercept. Circles indicate abnormal EEG records. Square indicates a baby where the CFOE was abnormal (0.44).

losses of measurements were again due to equipment failure and difficulty in gaining access to patients with severe hypotension. PBF decreased with decrease in MBP (Spearman's rho: $0.56, p=0.036, n=14$ ) (Fig. 4) and was abnormally low at MBP levels below $33 \mathrm{~mm} \mathrm{Hg}$. Low levels of PBF were associated with normal CFOE in all infants except for the one infant (case no. 5) described in more detail below (Fig. 5).

One female infant (case no. 5) of 26 wk gestation and birth weight $612 \mathrm{~g}$ (below 10th centile) had a normal EEG at a MBP level of $20 \mathrm{~mm} \mathrm{Hg}$. Her PBF was abnormally low (2.68 $\mathrm{mL} / 100 \mathrm{~mL} / \mathrm{min}$ ) and the CFOE (0.44) was abnormally high (Fig. 4).

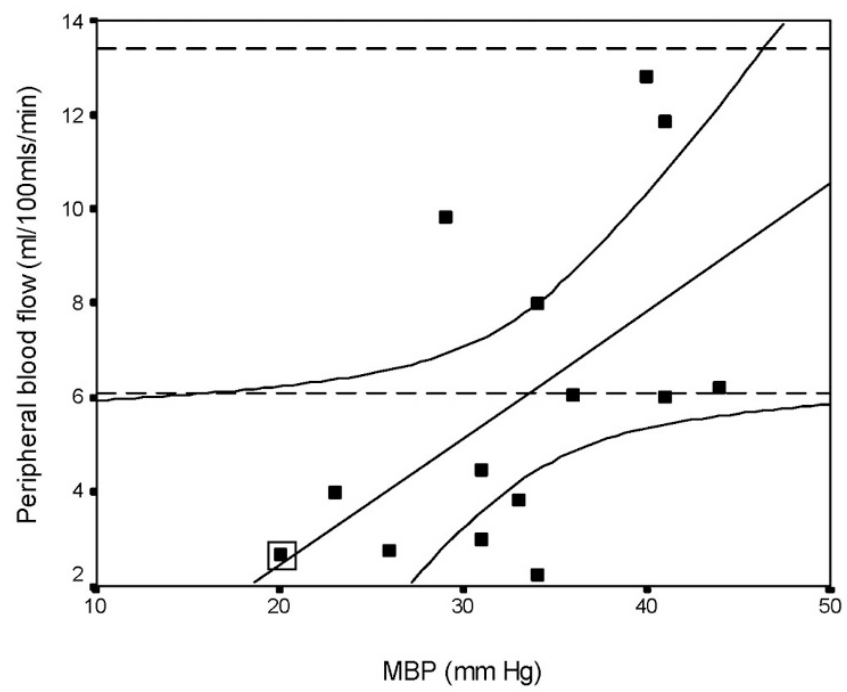

Figure 4. Relationship between $\mathrm{PBF}$ and $\mathrm{MBP}(n=14$; rho $=0.56$; $p=$ 0.036). Dotted lines show normal ranges of peripheral blood flow. Straight lines indicate regression line with $95 \%$ confidence intervals. Square indicates a baby where the CFOE was abnormal (0.44). 


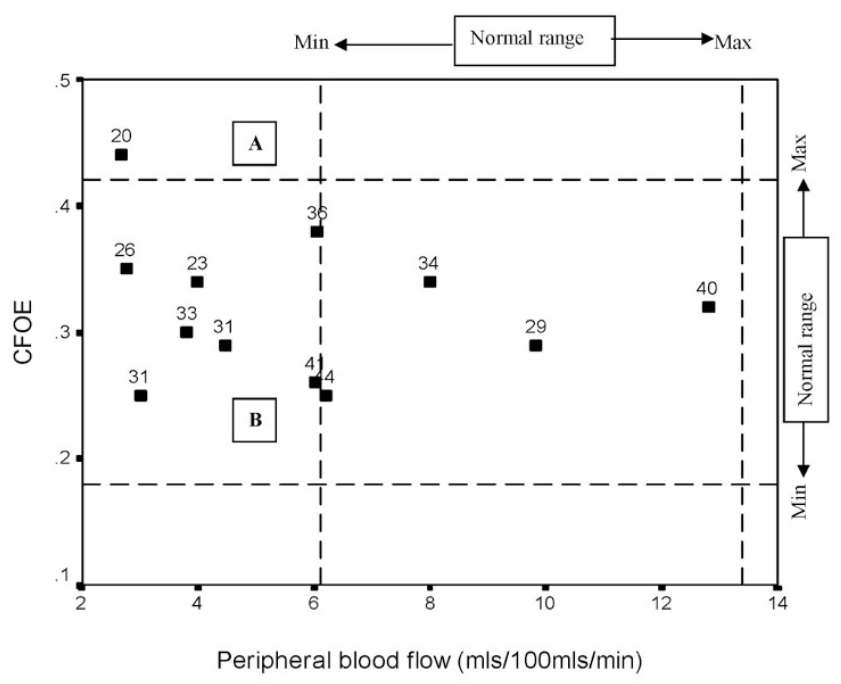

Figure 5. Relationship between MBP, PBF, and CFOE. Numbers next to points indicate $\mathrm{MBP}(\mathrm{mm} \mathrm{Hg})$. Infants with low $\mathrm{PBF}[<6.1 \mathrm{~mL} / 100 \mathrm{~mL} / \mathrm{min}$ (25)] mostly have normal CFOE at MBP range of $23-33 \mathrm{~mm} \mathrm{Hg}$ (quadrant B). One infant (case no. 5) with MBP of $20 \mathrm{~mm} \mathrm{Hg}$ and low PBF had high CFOE (quadrant A)

Sixteen infants received dopamine infusions [20 $\mu \mathrm{g} / \mathrm{kg} / \mathrm{min}$ $(n=4), 15 \mu \mathrm{g} / \mathrm{kg} / \mathrm{min}(n=1), 10 \mu \mathrm{g} / \mathrm{kg} / \mathrm{min}(n=8), 5$ $\mu \mathrm{g} / \mathrm{kg} / \mathrm{min}(n=3)]$. Of these 16 infants, two infants were also treated with dobutamine and one received dobutamine and hydrocortisone. Use of dopamine did not affect any of the outcome variables and was consistently excluded by the multiple regression analyses.

None of the cranial ultrasound scans on any of the 35 infants showed persistent echogenicity or cystic periventricular leukomalacia. The cranial ultrasound scans of the four infants with abnormal EEG records are described in Table 2. One infant with an abnormal EEG (case no. 2 in Table 2) showed gross ventricular dilatation on later cranial ultrasound scans.

Of the remaining 31 infants with normal EEG, 26 infants had normal or subependymal hemorrhages on later cranial ultrasound scans. Four infants developed intraventricular hemorrhages and one other case developed bilateral intraventricular hemorrhage with parenchymal extension during the third and fourth day after birth. Later ultrasound scans showed that two of these infants developed mild ventricular dilatation (rate of increase in head circumference measurements was along the centiles for the normal population) and hydrocephalus in one other case.

EEG was abnormal in five infants on d 3 and 4. The five EEG abnormalities were related to development of intraventricular hemorrhage $(n=4)$ and hypercarbia $(n=1)$ and not to MBP.

\section{DISCUSSION}

There is variation in the clinical management of hypotension in very low birth weight infants. The recommendation of the British Association of Perinatal Medicine that MBP levels in $\mathrm{mm} \mathrm{Hg}$ should not fall below the gestational age in weeks is a consensus opinion, which is unsupported by scientific evidence (23). The other common practice of maintaining MBP levels above $30 \mathrm{~mm} \mathrm{Hg}$ using volume expansion and inotropes is based on statistical associations between MBP levels below $30 \mathrm{~mm} \mathrm{Hg}$ and periventricular hemorrhage, cerebral ischemic lesions, and death (1-3). A third approach is to maintain the MBP above the 10th centile of a normal range based on birth weight or gestation and postnatal age $(3,22)$.

In this study, the level of MBP below which cerebral electrical activity became abnormal was found to be $23 \mathrm{~mm}$ $\mathrm{Hg}$. At MBP levels between 23 and $33 \mathrm{~mm} \mathrm{Hg}$, PBF was abnormally low while EEG patterns and CFOE remained normal. The EEG abnormalities in most (three out of four) of these babies whose MBP was below $23 \mathrm{~mm} \mathrm{Hg}$ during the first $48 \mathrm{~h}$ after birth were transient and none developed cystic periventricular leukomalacia. Even at a MBP below $23 \mathrm{~mm}$ $\mathrm{Hg}$, one infant maintained a normal EEG, but CFOE was increased, implying decreased cerebral oxygen delivery. The increases in MBP (22) and relative power of delta band (20) and decreases in CFOE (24) and $\mathrm{P}_{90}$ interburst interval (24) that are reported in Table 3 followed a similar pattern to that reported by other studies.

The observation that normal cerebral electrical activity is maintained at MBP levels below $30 \mathrm{~mm} \mathrm{Hg}$ is consistent with other studies. Tyszcuk et al. (8) showed that infants between 24 and $34 \mathrm{wk}$ gestation undergoing neonatal intensive care were able to maintain normal cerebral blood flow at a MBP range of 23.7-39.3 $\mathrm{mm} \mathrm{Hg}$. Munro et al. (25) showed that in 12 hypotensive (MBP: $25 \pm 1 \mathrm{~mm} \mathrm{Hg}$ ) extremely low birth weight infants also maintained normal cerebral blood flow (14 $\pm 1 \mathrm{~mL} / 100 \mathrm{~g} / \mathrm{min}$ ). Pryds and Greisen (26) recorded singleflash visual evoked potentials in 32 preterm infants (mean gestational age: 29 wk) during the first day of life. Amplitude and latency of the signal were unchanged during episodes of low cerebral blood flow and correspondingly low cerebral oxygen delivery and severe arterial hypotension (MBP: 10 $\mathrm{mm} \mathrm{Hg}$ ), provided that the arterial oxygen tension was greater than $5 \mathrm{kPa}(26)$.

In this study, the EEG did not become normal at the same time as the recovery of MBP. The cellular and biochemical events that underlie the recovery of EEG following anoxia are not well understood. Restoration of circulation and substrate supply are necessary for repletion of cellular ATP stores and return to normal levels of $\mathrm{pH}$ and ion concentrations. MacMillan (27) reported that EEG recovered in parallel with the recovery of Na-K-ATPase activity over $72 \mathrm{~h}$ after global ischemia in rat forebrain. Thus, although metabolic recovery is necessary for electrophysiological recovery, the two are not necessarily contemporaneous and there are numerous experimental models in which the EEG recovers more slowly than cerebral energy metabolism, as reflected by brain oxygen consumption, high-energy phosphate content, and intracellular $\mathrm{pH}(28)$.

As observed in a previous study, PBF diminished with decreasing systemic MBP (16). Abnormally low levels of PBF were measured at MBP levels between 23 and $33 \mathrm{~mm} \mathrm{Hg}$, although simultaneous measurements of CFOE were normal. Two inferences can be drawn from these observations. First, that peripheral perfusion diminishes at MBP levels higher than 
the critical level of MBP below which cerebral electrical activity becomes abnormal. Second, that in these infants cerebral oxygen delivery is maintained at MBP levels between 23 and $33 \mathrm{~mm} \mathrm{Hg}$, probably by diminished peripheral perfusion.

The critical level of MBP below which permanent brain damage occurs is of interest to clinicians. Watanabe et al. (29) showed that $95 \%$ of infants with normal EEG patterns had normal neurodevelopmental outcome. The remaining $5 \%$ of infants were able to walk and exhibited only minimal signs of cerebral palsy (29). It may therefore be reasonable to assume that preservation of normal EEG between MBP levels of 23 and $30 \mathrm{~mm} \mathrm{Hg}$ indicates normal cerebral perfusion. However, the prolonged periods of electrical discontinuity that were noted in relation to MBP levels below $23 \mathrm{~mm} \mathrm{Hg}$ have been associated with adverse neurologic outcome (12). Nevertheless, the EEG abnormalities noted in this study do not necessarily represent evidence of irreversible brain damage because the EEG abnormalities recovered and became normal by the fourth day in most infants. In the only infant that the EEG abnormality did not recover (case no. 2 in Table 2), there was an intraventricular hemorrhage and the persistent EEG abnormality may have been a consequence of the extensive nature of this hemorrhage. It should be noted that none of the infants studied developed cystic periventricular leukomalacia.

There are two methodological concerns with this study. First, the relationship between low MBP and abnormal cerebral electrical activity remains an association. However, several other variables (Table 1) that may cause EEG abnormalities were considered and hypotension remained the single most prominent cause. Second, because the management of hypotension was left to the clinical team, only five infants with seriously low MBP below $23 \mathrm{~mm} \mathrm{Hg}$ could be studied. The results of the present study cannot therefore be interpreted as sufficient support for a recommendation that MBP levels between 23 and $30 \mathrm{~mm} \mathrm{Hg}$ do not require treatment. However, the results of this study generate hypotheses that need to be further addressed. They raise the possibility that these immature infants may maintain cerebral perfusion at lower levels of systemic blood pressure than has hitherto generally been thought to be the case.

In only one infant was there a lack of agreement between the qualitative EEG reporting and quantitative methods: the EEG recording was reported by the neurologist to show low amplitude slow wave activity (Table 2, case no. 2, d 1), but the quantitative analysis was normal. This lack of agreement was probably due to the poor sensitivity of spectral analysis in detecting this moderately abnormal EEG: a combination of slowing of the EEG waveforms (which would increase the relative power of the delta band) and a decrease in their amplitude (which would decrease the relative power of the delta band) may have resulted in little or no net change in the relative power of delta band.

In conclusion, cerebral electrical activity remained normal at MBP levels between 23 and $30 \mathrm{~mm} \mathrm{Hg}$ and was found to be abnormal at MBP levels below $23 \mathrm{~mm} \mathrm{Hg}$. Cerebral electrical activity and CFOE were maintained at lower MBP levels than currently accepted by most clinicians.

\section{REFERENCES}

1. Bada HS, Korones SB, Perry EH, Arheart KL, Ray JD, Pourcyrous M, Magill HL, Runyan W Somes, GW, Clark 3rd FC 1990 Mean arterial blood pressure changes in premature infants and those at risk for intraventricular hemorrhage. J Pediatr 117:607-614

2. Miall-Allen VM, De Vries LS, Whitelaw AG 1987 Mean arterial blood pressure and neonatal cerebral lesions. Arch Dis Child 62:1068-1069

3. Watkins AM, West CR, Cooke RW 1989 Blood pressure and cerebral haemorrhage and ischaemia in very low birthweight infants. Early Hum Dev 19:103-110

4. Greisen G, Trojaborg W 1987 Cerebral blood flow, $\mathrm{PaCO}_{2}$ changes, and visual evoked potentials in mechanically ventilated, preterm infants. Acta Paediatr Scand 76:394-400

5. Jorch G, Jorch N 1987 Failure of autoregulation of cerebral blood flow in neonates studied by pulsed Doppler ultrasound of the internal carotid artery. Eur J Pediatr $146: 468-472$

6. Koyama K, Mito T, Takashima S, Suzuki S 1990 Effects of phenylephrine and dopamine on cerebral blood flow, blood volume, and oxygenation in young rabbits. Pediatr Neurol 6:87-90

7. Lou HC, Lassen NA, Friis-Hansen B 1979 Impaired autoregulation of cerebral blood flow in the distressed newborn infant. J Pediatr 94:118-121

8. Tyszczuk L, Meek J, Elwell C, Wyatt JS 1998 Cerebral blood flow is independent of mean arterial blood pressure in preterm infants undergoing intensive care. Pediatrics 102:337-341

9. Greisen G, Pryds O 1989 Low CBF, discontinuous EEG activity, and periventricular brain injury in ill, preterm neonates. Brain Dev 11:164-168

10. Scher MS 1999 Electroencephalography of the newborn: normal and abnormal features. In: Niedermeyer E, Da Silva FL (eds) Electroencephalography: Basic Principles, Clinical Applications, and Related Fields. Williams \& Wilkins, Baltimore, pp 896-946

11. Hayakawa M, Okumura A, Hayakawa F, Watanabe K, Ohshiro M, Kato Y, Takahashi R, Tauchi N 2001 Background electroencephalographic (EEG) activities of very preterm infants born at less than 27 weeks gestation: a study on the degree of continuity. Arch Dis Child Fetal Neonatal Ed 84:F163-F167

12. Marret S, Parain D, Menard JF, Blanc T, Devaux AM, Ensel P, Fessard C, Samson-Dollfus D 1997 Prognostic value of neonatal electroencephalography in premature newborns less than 33 weeks of gestational age. Electroencephalogr Clin Neurophysiol 102:178-185

13. Ichord RN, Kirsch JR, Koehler RC, Traystman RJ 1999 Cerebral anoxia: experimental view. In: Niedermeyer E, Da Silva FL (eds) Electroencephalography: Basic Principles, Clinical Applications, and Related Fields. Williams \& Wilkins, Baltimore, pp 432-444

14. Wardle SP, Yoxall CW, Weindling AM 2000 Determinants of cerebral fractional oxygen extraction using near infrared spectroscopy in preterm neonates. J Cereb Blood Flow Metab 20:272-279

15. Yoxall CW, Weindling AM, Dawani NH, Peart I 1995 Measurement of cerebral venous oxyhemoglobin saturation in children by near-infrared spectroscopy and partial jugular venous occlusion. Pediatr Res 38:319-323

16. Wardle SP, Yoxall CW, Weindling AM 1999 Peripheral oxygenation in hypotensive preterm babies. Pediatr Res 45:343-349

17. Parmelee AH Jr.,Wenner,WH, Akiyama Y, Schultz M, Stern E 1967 Sleep states in premature infants. Dev Med Child Neurol 9:70-77

18. Jasper HH 1958 The Ten - Twenty Electrode System of the International Federation. Electroencephalogr Clin Neurophysiol 10:371-375

19. Biagioni E, Bartalena L, Biver P, Pieri R, Cioni G 1996 Electroencephalographic dysmaturity in preterm infants: a prognostic tool in the early postnatal period. Neuropediatrics 27:311-316

20. Victor S, Appleton RE, Beirne M, Marson AG, Weindling AM 2005 Spectral analysis of electroencephalography in premature newborn infants: normal ranges. Pediatr Res 57:336-341

21. Wardle SP, Yoxall CW, Crawley E, Weindling AM 1998 Peripheral oxygenation and anemia in preterm babies. Pediatr Res 44:125-131

22. Cunningham S, Symon AG, Elton RA, Zhu C, McIntosh N 1999 Intra-arterial blood pressure reference ranges, death and morbidity in very low birthweight infants during the first seven days of life. Early Hum Dev 56:151-165

23. 1992 Development of audit measures and guidelines for good practice in the management of neonatal respiratory distress syndrome. Report of a Joint Working Group of the British Association of Perinatal Medicine and the Research Unit of the Royal College of Physicians. Arch Dis Child 67:1221-1227

24. Kissack CM, Garr R, Wardle SP, Weindling AM 2004 Cerebral fractional oxygen extraction in very low birth weight infants is high when there is low left ventricular output and hypocarbia but is unaffected by hypotension. Pediatr Res 55:400-405

25. Munro MJ, Walker AM, Barfield CP 2004 Hypotensive extremely low birth weight infants have reduced cerebral blood flow. Pediatrics 114:1591-1596

26. Pryds O, Greisen G 1990 Preservation of single-flash visual evoked potentials at very low cerebral oxygen delivery in preterm infants. Pediatr Neurol 6:151-158

27. MacMillan V 1982 Cerebral $\mathrm{Na}+, \mathrm{K}+$-ATPase activity during exposure to and recovery from acute ischemia. J Cereb Blood Flow Metab 2:457-465

28. Rosen I, Smith ML, Rehncrona S 1984 Quantitative EEG and evoked potentials after experimental brain ischemia in the rat; correlation with cerebral metabolism and blood flow. Prog Brain Res 62:175-183

29. Watanabe K, Hayakawa F, Okumura A 1999 Neonatal EEG: a powerful tool in the assessment of brain damage in preterm infants. Brain Dev 21:361-372 